\title{
The role of fast and slow EEG activity during sleep in males and females with major depressive disorder
}

\author{
PHILIP CHENG, JENNIFER GOLDSCHMIED, PATRICIA DELDIN, ROBERT HOFFMANN, AND \\ ROSEANNE ARMITAGE \\ Department of Psychiatry, University of Michigan, Ann Arbor, Michigan, USA
}

\begin{abstract}
Sleep difficulties are highly prevalent in depression, and appear to be a contributing factor in the development and maintenance of symptoms. However, despite the generally acknowledged relationship between sleep and depression, the neurophysiological substrates underlying this relationship still remain unclear. Two main hypotheses were tested in this study. The first hypothesis states that sleep in depression is characterized by inadequate generation of restorative sleep, as indexed by reduced amounts of slow-wave activity. Conversely, the second hypothesis states that poor sleep in depression is due to intrusions of fast-frequency activity that may be reflective of a hyperaroused central nervous system. This study aimed to test both hypotheses in a large sample of individuals with clinically validated depression, as well as to examine sex as a moderator. Results suggest that depression is better characterized by an overall decrease in slow-wave activity, which is related to elevated anxious and depressed mood the following morning. Results also suggest that females may be more likely to experience fast frequency activity related to depression symptom severity.
\end{abstract}

Descriptors: Sleep, Psychopathological, EEG, Depression

Anecdotal and scientific evidence indicate that cognitive and emotional functioning can be compromised by poor sleep occurring the previous night (Barnett \& Cooper, 2008). Not surprisingly, sleep disturbances such as difficulty initiating/maintaining sleep or restless sleep are reported in almost all mental illnesses (Dolsen, Asarnow, \& Harvey, 2014; Harvey, 2008). Sleep difficulties are especially relevant in major depressive disorder (MDD), with over $80 \%$ of patients with depression reporting poor quality sleep linked with adverse consequences for mood and daytime functioning (Reynolds \& Kupfer, 1987; Vanderlind et al., 2014). However, despite general acknowledgement of the intimate relationship between sleep and depression, the specific neurophysiological pathways associated with mood dysregulation in depression still remain unclear. Understanding how sleep is associated with mood disturbances in depression is important because it may inform intervention targets (Manber et al., 2008), and perhaps even reduce risk for future episodes (Reynolds et al., 1997).

Among various mechanisms that are implicated in disturbed sleep in depression, impaired sleep drive and increased arousal have received the most attention. Although related, impaired sleep drive

This study was supported by an NIMH Grant (R01 MH061515) awarded to RA. The authors would also like to thank the University of Michigan Department of Psychology, Department of Psychiatry, and the Depression Center for their continued support.

Address correspondence to: Philip Cheng, Ph.D., Sleep Disorders and Research Center, Henry Ford Hospital, Detroit, MI 48202, USA. E-mail: pcheng1@hfhs.org and increased arousal are not necessarily inverses of the same phenomenon, and may therefore implicate differential neurophysiological substrates. On the one hand, depressed individuals may exhibit inadequate generation of restorative sleep as indexed by reduced amounts of slow-wave (i.e., delta) activity (Armitage, 2007; Borbély et al., 1984), which results in impaired daytime functioning. Slow-wave activity has been associated with deeper and better quality sleep, and may play an important role in restoration on various levels, including synaptic homeostasis as well as metabolic regulation (Tononi \& Cirelli, 2006). Alternatively, depressed individuals may experience physiological hyperarousal as indexed by intrusions of fast frequency activity (i.e., beta activity; Nofzinger et al., 1999; Riemann et al., 2010). Fast frequency activity has commonly been associated with cortical arousal, and has been widely implicated in insomnia (Mendelson, James, Garnett, Sack, \& Rosenthal, 1986; Riemann et al., 2010; Stepanski, Zorick, Roehrs, \& Young, 1988), which is highly comorbid with depression. Cortical hyperarousal has often been observed during early portions of the night (Perlis, Merica, Smith, \& Giles, 2001), and is posited to relate to difficulties transitioning to and maintaining a sleep state, thereby leading to daytime impairments such as fatigue. Due to the high comorbidity between insomnia and depression, it has also been proposed that hyperarousal may similarly explain poor quality sleep and its daytime consequences in depression (Armitage \& Hoffmann, 1997; Hall et al., 2000; Mendelson et al., 1987; Nofzinger et al., 1999), especially in females (Armitage \& Hoffmann, 2001; Armitage, Hudson, Trivedi, \& Rush, 1995). The differentiation of these two physiological processes may further characterize the relationship 
between sleep and depression, which can also potentially inform refinement of interventions.

Sex differences in depression has been widely documented, and includes the disproportion of prevalence, expression (e.g., internalizing vs. externalizing behaviors), and experience of symptoms (e.g., increased rumination in females; Nolen-Hoeksema, 1990). Extant research also indicates that brain physiology during sleep in depression differs by sex (Armitage, 2007; Reynolds et al., 1990). Compared to women with MDD, men with MDD show significantly lower amounts of slow-wave activity at the beginning of the night, with a slower rate of decay of slow-wave activity throughout the night (Armitage, Hoffmann, Fitch, Trivedi, \& Rush, 2000; Frey et al., 2012; Goldschmied, Cheng, Armitage, \& Deldin, 2014; Plante et al., 2012). This suggests that the sleep neurophysiology of depression in men may differ from women, with men showing a tendency toward a less responsive sleep homeostatic system. Prior research has also proposed that hyperarousal may be more pronounced in women (Armitage et al., 1995). This may be attributable to the positive association between cortical hyperarousal and rumination, which is characteristic of depression in females. Together, these sex differences indicate that the ways in which sleep abnormalities contribute to depression may involve disparate neurophysiological substrates, and may therefore respond differently to the interventions.

Additionally, while studies have sought to delineate ways in which sleep in depression differs from that in healthy individuals, we are only beginning to understand how these differences relate to the experience of depression. For example, while abnormalities in slow-wave activity have been implicated in depression (e.g., Goldschmied et al., 2014), little is known regarding how these abnormalities are associated with specific dimensions of depression, such as mood or somatic symptoms, or even if the relationship can be described linearly. Given the heterogeneity of symptom presentation in MDD, a dimensional characterization of the relationship between sleep and depression may lead to increased specificity in interventions. One earlier study described linear relationships between dimensions of depression symptoms and sleep variables (Perlis, Giles, Buysse et al., 1997). This study relied on visual stage scoring and subsequent period amplitude analysis, showing that average counts of delta waves per minute during NREM (nonrapid eye movement) periods were associated with dimensions of depression. However, though the differentiation between increased arousal versus impaired sleep drive was discussed, the focus on delta counts in NREM sleep in this study precluded the direct comparison of the two mechanisms. Furthermore, the use of visually scored stages to organize sleep data becomes somewhat redundant with subsequent quantitative EEG analysis, as stage scoring relies in part on a visual analysis of underlying EEG frequency structures (i.e., NREM sleep is designate based on visual detection of delta activity).

In order to address the current gaps in research, this study aimed to (a) further characterize the respective roles of slow and fast frequency activity during sleep in depression, (b) explore its relationship with symptom severity and mood, and (c) test sex as a moderator in these relationships. A quantitative EEG approach was utilized across the sleep period, organized by hour of sleep to avoid preanalysis clustering of EEG data by its underlying frequency structures. EEG activity was quantified from slow to fast frequency bins using period spectral analysis, and compared males and females in the MDD and healthy control groups. If depression is characterized by dysregulations in homeostatic sleep drive, group comparisons would reveal differences in slower frequency activity (delta and theta bands). If depression is instead characterized by hyperarousal, group comparisons would reveal differences in higher frequency activity (beta, sigma, or alpha bands). A more dimensional approach was also utilized in order to characterize the linearity and strength of the relationship between neurophysiology during sleep and depression severity. Variables of interest were reduced and refined based in part on results from the previous group comparison and submitted to a linear regression, with depression symptom severity as the outcome variable and sex as a moderator. It was hypothesized that sex would moderate the relationship between slow and fast frequency activity and depression severity. In particular, depression in males may be more related to deficits in slow frequency activity, whereas depression in females may be more associated with intrusions of fast frequency activity. Finally, exploratory correlations were conducted with sleep variables and mood.

\section{Method}

The sample was extracted from archival sleep data collected between 1991 and 2011 from large academic institutions in Texas and Michigan. All studies were conducted for the same general purpose: to examine the relationship between quantitative sleep EEG and depression. All studies followed standardized procedures across sleep nights. Analyses did not reveal any group differences by data collection site. All participants were recruited for sleep studies through flyers posted in the community. Participants all received a telephone screen, which was then followed with an inperson Structured Clinical Interview for the Diagnostic and Statistical Manual of Mental Disorders (First, Spitzer, Gibbon, \& Willams, 2002). The SCID for the DSM-III or DSM-IV was administered depending on date of recruitment. A detailed personal and family history was collected at the clinical interview. Exclusionary criteria included shift work, independent sleep disorders (e.g., obstructive sleep apnea, narcolepsy, bruxism, and periodic limb movement disorder), and any medication use within the previous 4 weeks, with the exception of nonsteroidal antiinflammatory drugs.

All healthy control participants were medically fit and had no personal or family history of Axis I disorders based on the SCID. Depressed participants were physically healthy, but met DSM-III or DSM-IV criteria for a current major depressive episode without psychosis. Clinical interviews were conducted by doctoral level clinicians, clinically trained and supervised graduate students, or licensed and trained social workers. Hamilton Depression Rating Scale (HDRS; Hamilton, 1967) scores were also completed in order to measure severity of depression symptoms. A total of 158 participants ( 80 with MDD) were selected based on age (18 to 45), sex, and viable records from all relevant sleep studies. Demographic and sleep variables are included in Table 1.

Once enrolled in the sleep study, all participants maintained their own habitual, but regularized sleep schedule and recorded sleep diaries 5 days prior to the first night of the sleep study. Actigraphy data, based on physical movement and light exposure, were also collected to confirm the accuracy of the sleep diary records and regularity of sleep prior to lab recordings. The first night in the lab served as adaptation to the instrumentation and environment. All data reported here are based on the second night in the lab. 
Table 1. Demographic and Sleep Variables

\begin{tabular}{|c|c|c|c|c|c|}
\hline \multirow[b]{2}{*}{ Variables } & \multicolumn{2}{|c|}{ MDD } & \multicolumn{2}{|c|}{$\mathrm{HC}$} & \multirow{2}{*}{$\begin{array}{l}\text { Diagnosis } \\
p \text { value }\end{array}$} \\
\hline & Males & Females & Males & Females & \\
\hline$N$ & 35 & 47 & 42 & 36 & .21 \\
\hline Age & $32.4(7.0)$ & $29.0(6.3)$ & $28.6(5.7)$ & $28.7(5.8)$ & .07 \\
\hline HDRS & $21.4(4.4)$ & $22.9(4.2)$ & $0.7(1.1)$ & $0.9(1.4)$ & $<.001$ \\
\hline$\% \mathrm{~S} 1$ & 10.9 (7.9) & $5.8(7.5)$ & $6.7(5.8)$ & $5.9(5.0)$ & .002 \\
\hline$\% \mathrm{~S} 2$ & $53.1(8.9)$ & $52.3(8.6)$ & $55.3(8.2)$ & $53.1(7.8)$ & .02 \\
\hline$\% \mathrm{~S} 3$ and 4 & $8.2(8.8)$ & $12.0(10.2)$ & $6.9(8.3)$ & $12.9(8.5)$ & .36 \\
\hline$\% R$ & $22.0(6.2)$ & $22.0(6.3)$ & $22.8(6.2)$ & $23.0(5.4)$ & .53 \\
\hline Hours in bed & $7.1(0.6)$ & $6.8(0.6)$ & $6.8(0.2)$ & $6.8(0.2)$ & .21 \\
\hline SOL (min) & $11.0(14.2)$ & $16.4(12.1)$ & $7.4(8.5)$ & $9.2(13.6)$ & .003 \\
\hline $\operatorname{Beta}\left(\mu V^{2}\right)$ & $23.1(4.9)$ & $26.3(8.3)$ & $23.6(4.4)$ & $24.7(5.3)$ & .39 \\
\hline Sigma $\left(\mu \mathrm{V}^{2}\right)$ & $55.9(15.0)$ & $65.2(16.5)$ & $60.1(15.7)$ & $63.5(18.4)$ & .85 \\
\hline Alpha $\left(\mu \mathrm{V}^{2}\right)$ & $93.4(24.0)$ & $104.6(26.2)$ & $106.2(27.5)$ & $105.3(32.1)$ & .17 \\
\hline Theta $\left(\mu \mathrm{V}^{2}\right)$ & $88.4(19.2)$ & $99.2(21.3)$ & $100.7(21.4)$ & $103.3(24.2)$ & .03 \\
\hline Delta $\left(\mu \mathrm{V}^{2}\right)$ & $334.9(68.0)$ & $367.7(70.7)$ & $376.4(74.3)$ & $394.9(89.4)$ & .01 \\
\hline
\end{tabular}

Note. $\mathrm{MDD}=$ major depressive disorder; $\mathrm{HC}=$ healthy controls; HDRS = Hamilton Depression Rating Scale; SOL = sleep onset latency.

\section{Signal Processing}

Participants' sleep EEG were recorded on either a four or eight EEG electrode montage, including leg leads, chest respiration band, and a nasal-oral thermistor. The eight EEG montage included electrodes at the left and right frontal, central, parietal, and occipital areas (F3, F4, C3, C4, P3, P4, O1, O2), whereas the four EEG montage included central and parietal areas (C3, C4, P3, P4). First night polysomnography recording was used to rule out any suspected sleep disordered breathing, periodic limb movements, bruxism, or other independent sleep disorders. Bipolar chin-cheek electromyograms (EMGs) were also recorded. EEG electrodes were referenced to the left and right ear lobes linked together through a $10 \mathrm{kOhm}$ resistor.

One hundred and forty-six records were acquired on a GRASS P511 amplifier-based paperless polygraph, and 14 records were collected on a Vitaport III digital data acquisition system. Data systems were cross-validated in two ways. First, a sine wave generator was used for signal recording on both data acquisition systems simultaneously, and then subjected to power spectral analysis to ensure that spectral profiles were identical. Second, all night EEG data were acquired simultaneously from 10 subjects and analyzed to ensure that spectral power values fell within the $95 \%$ confidence interval of each system.

EEG was recorded at the equivalent sensitivity of $5(50 \mu \mathrm{V}$, 0.5 -s calibration), corresponding to a gain of 50,000, with bandpass filters set to the equivalent of 0.3 and $30 \mathrm{~Hz}$, respectively. Signals were digitized online at $256 \mathrm{~Hz}(62.5 \mathrm{~Hz}$ for electrooculogram and EMG) and displayed digitally during acquisition. All raw digitized data were stored on mass media.

\section{Behavioral Measures}

All participants completed the HDRS, and a subset of depressed participants $(N=36)$ also reported their mood following sleep via the Profile for Mood States questionnaire (POMS; (McNair, Lorr, \& Droppleman, 1971), and a postsleep questionnaire that measured perceived sleep onset latency, wake after sleep onset, time in bed, time asleep, as well as visual analogue scales rating the quality of sleep, restfulness upon awakening, and sleepiness upon awakening.

\section{Data Analyses}

Sleep was stage-scored on an epoch-by-epoch basis according to Rechtschaffen and Kales (1968) criteria by trained technicians with $90 \%$ interrater reliability, who were blind to the diagnostic status of the participants. Power spectral analysis (PSA) quantified EEG in 30-s segments and summed across the following frequency categories: delta $(0.5 \mathrm{~Hz}$ to $3.9 \mathrm{~Hz})$, theta $(4 \mathrm{~Hz}$ to $7.9 \mathrm{~Hz})$, alpha $(8 \mathrm{~Hz}$ to $11.9 \mathrm{~Hz}$ ), sigma (12 Hz to $14.9 \mathrm{~Hz}$ ), and beta ( $15 \mathrm{~Hz}$ to $30 \mathrm{~Hz}$ ). Analyses also quantified EEG by increments of $1 \mathrm{~Hz}$ for a finer analysis (see Figure 1). The resulting power values (power under the curve, expressed in $\mu \mathrm{V}^{2}$ ) were then averaged within each epoch (30 s) in order to match epoch conventions for visual sleep stage scoring. This was completed in order for exclusion of all epochs marked with movement or arousal to remove artifacts from analyses. As such, only epochs from stages 1, 2, 3, 4, and REM were included for analysis. Instead of organizing sleep by visually scored sleep stages, which already divide sleep using the underlying frequency structure, analyses of sleep were organized by hour of sleep

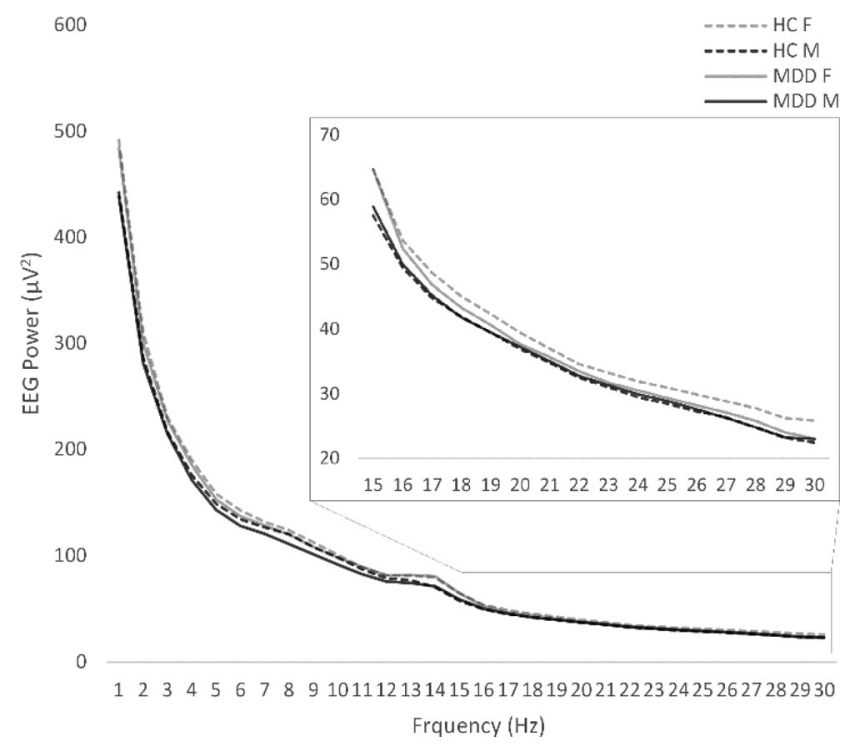

Figure 1. All-night frequency spectra (average of $\mathrm{C} 3$ and $\mathrm{C} 4$ ) by $1 \mathrm{~Hz}$ increments. 

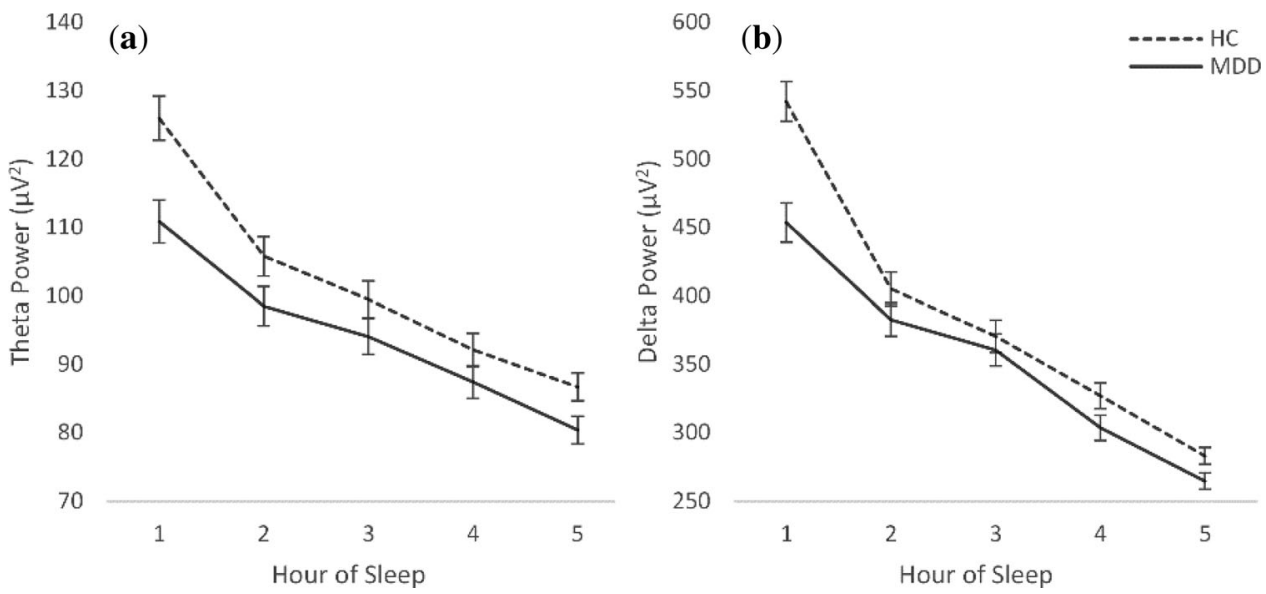

Figure 2. a: Theta power across the hours of the night. b: Delta power across the hours of the night. Hour 5 represents average activity in 5th and remaining hours of the night. Participants did not have equal total sleep times, and the least common factor $(5 \mathrm{~h})$ was used to preserve sample size.

to facilitate an all-night quantitative analysis of the sleep EEG Because central electrodes were recorded in all participants, analyses were conducted using data from $\mathrm{C} 3$ and $\mathrm{C} 4$ in order to maximize sample size. Natural log transformations of absolute power were completed for all variables that showed skewness greater than \pm 1 or kurtosis greater than 3 .

The EEG data were submitted to a repeated measures analysis of covariance (ANCOVA), with frequency (delta, theta, alpha, sigma, and beta), laterality (C3 and C4), hour of sleep (hours 1 through 5), sex (male, female), and group (healthy control, and MDD) as independent variables, with age as a covariate. The Greenhouse-Geisser correction was utilized as a correction for violations of sphericity. Partial eta squared $\left(\eta_{\mathrm{p}}{ }^{2}\right)$ was reported as a measure of effect size. Follow-up analyses were also conducted on a subset of participants for whom frontal electrodes were also recorded $(N=110 ; \mathrm{MDD}=36)$. Statistical analyses were conducted in SPSS version 22 (IBM Corp., Armonk, NY).

In order to further investigate the relationship between EEG characteristics and depression severity using a dimensional approach, a linear regression with HDRS scores entered as the dependent variable was completed. EEG predictors were reduced in order to address issues of collinearity, and refined based upon results from the repeated measures ANCOVA. EEG variables were reduced by averaging left and right central electrodes for high (beta and sigma) and low (delta and theta) frequency groups. Finally, correlations between POMS subscales and sleep EEG variables were also conducted.

\section{Results}

\section{Group Differences in EEG Power by Frequency}

The repeated measures five-way Frequency $\times$ Laterality $\times$ Hour of Sleep $\times$ Sex $\times$ Group ANCOVA indicated that EEG power differed between groups, with a significant frequency band and hour of sleep interaction, Frequency $\times$ Hour of Sleep $\times$ Group, $F(3.4,527.6)=$ $5.6, p=.001, \eta_{\mathrm{p}}{ }^{2}=.035$. As hypothesized, those with MDD showed significantly less power in the slow frequency bands relative to healthy controls (HCs), particularly in the first hour of the night, namely, in the theta, $F(1,155)=8.900, p=.003, \eta_{\mathrm{p}}{ }^{2}=.055$, and delta bands, $F(1,155)=15.366, p=.001, \eta_{\mathrm{p}}{ }^{2}=.090$ (see Figure 2). No group differences were detected in the beta frequency.

Group differences did not vary by sex, though females $(M=135.39, S E=2.78)$ across both groups showed higher overall
EEG power compared to males $(M=127.16, S E=2.85)$, sex, $F(1,153)=4.25, p=.04, \eta_{\mathrm{p}}{ }^{2}=.027$

\section{Frontal Beta Activity}

In order to further explore the cortical hyperarousal hypothesis, post hoc analyses for beta activity were also conducted in a subsample of 110 individuals (36 with MDD, 20 females; 74 HC, 34 females) for whom EEG from both frontal and central electrodes were acquired. This was completed for comparison to the studies in insomnia demonstrating increased fast frequency activity in the frontal sites. While a significant Region $\times$ Group effect was detected, $F(1,105)=10.56, p=.002, \eta_{\mathrm{p}}{ }^{2}=.09$, results did not indicate group differences in the frontal, group, $F(1,105)=.310$, $p=.58, \eta_{\mathrm{p}}{ }^{2}=.003$, or central regions, group, $F(1,105)=.642$, $p=.43, \eta_{\mathrm{p}}{ }^{2}=.003$. A nonsignificant trend was detected between frontal $(M=26.959, S E=1.331)$ and central $(M=22.695$, $S E=.861)$ regions in the MDD group, $F(1,33)=3.374, p=.08$, $\eta_{\mathrm{p}}{ }^{2}=.09$. No differences in region were detected in the HC group. Power analyses conducted post hoc using G*Power (Faul, Erdfelder, Lang, \& Buchner, 2007) revealed adequate power (.8) to detect group differences.

\section{Sex Differences in the Relationship Between Sleep EEG and HDRS Depression Severity}

In addition to examining group differences, analyses also explored the relationship between EEG characteristics and depression severity, as well as any sex differences in this relationship. As such, a hierarchical linear regression was performed with HDRS depression severity as the outcome measure. The model included age, slow frequency activity, fast frequency activity, sex, the interaction of slow frequency activity with sex, and the interaction of fast frequency activity with sex. The addition of age as a predictor was not significant and therefore was excluded from the final model. Collinearity was assessed using both tolerance and variance inflation factor statistics, and no issue of collinearity was detected.

Results (see Table 2) revealed that lower slow frequency activity (delta and theta) during the first hour significantly predicted higher HDRS scores, whereas fast frequency activity in the first hour (beta and sigma) did not significantly predict depression severity. However, results did indicate that the association between high frequency activity and depression severity varied by sex, with 
Table 2. Summary of Hierarchical Linear Regression for Variables Predicting HDRS

\begin{tabular}{lcrc}
\hline \hline Variables & $\mathrm{B}$ & \multicolumn{1}{c}{$S E$} & \multicolumn{1}{c}{$\beta$} \\
\hline Slow frequency (Hour 1) & -.027 & .008 & $-.327 * * *$ \\
Fast frequency (Hour 1) & -.011 & .068 & -.162 \\
Sex & 5.743 & 8.048 & -.023 \\
Slow Frequency (Hour 1) $\times$ Sex & -.011 & .012 & -.319 \\
Fast Frequency (Hour 1) $\times$ Sex & .168 & .086 & $.792 *$ \\
$F$ & $6.109 * * *$ & & \\
\hline \hline
\end{tabular}

Note. Sex $(0=$ Male).

$* p<.05, * * * p<.001$

only increased high frequency activity in females predicting increased depression severity. The differential slopes of this interaction are plotted in Figure 3.

\section{Mood in Depression, Postsleep Questionnaire, and Sleep EEG}

In addition to examining depression severity, analyses also explored how mood varied by sleep in a subsample of 34 participants with MDD, all of whom participated in the same study where mood data were collected. Due to the smaller sample size, the effect of sex was not examined in this sample. Initial correlations between depression symptom severity (HDRS) and mood data (POMS) revealed results in the expected directions, though these effects were small and did not reach statistical significance. This provided support that the POMS scores reflected disparate information from the HDRS. Correlations using the POMS questionnaire revealed that decreased delta activity across the night (indexed by activity averaged from the central leads) was related to increased anxiety as indexed by the tension-anxiety subscale $(r=-.434$, $p=.01)$ and increased confusion-bewilderment scores $(r=-.373$, $p=.03$; see Figure 4). Effects approaching statistical significance included increased depression-dejection scores with decreased delta activity $(r=-.290, p=.09)$, and increased tension-anxiety scores with decreased beta activity $(r=-.312, p=.07)$

Comparable to the correlations with mood, results from the postsleep questionnaire also revealed negative outcomes with

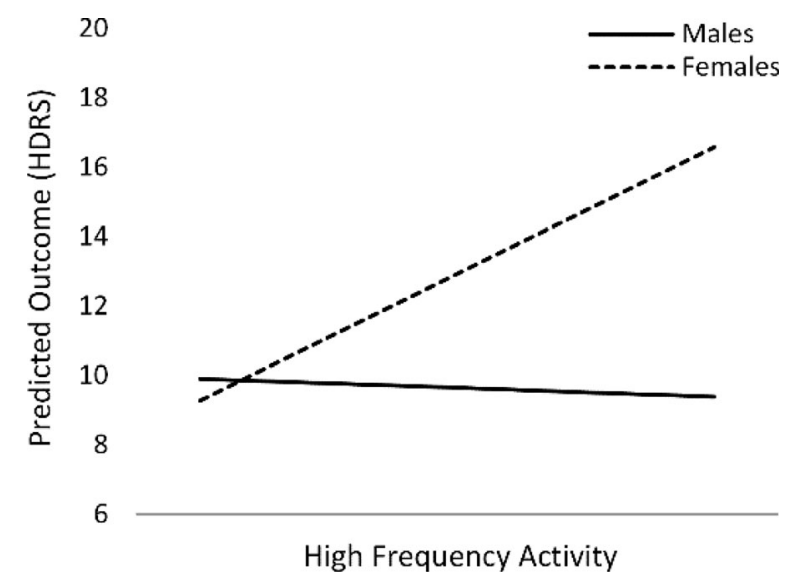

Figure 3. The differential slopes from the High Frequency $\times$ Sex interaction as a significant predictor of depression severity. High frequency activity is an average of beta and sigma activity from the central leads. HDRS $=$ Hamilton Depression Rating Scale.

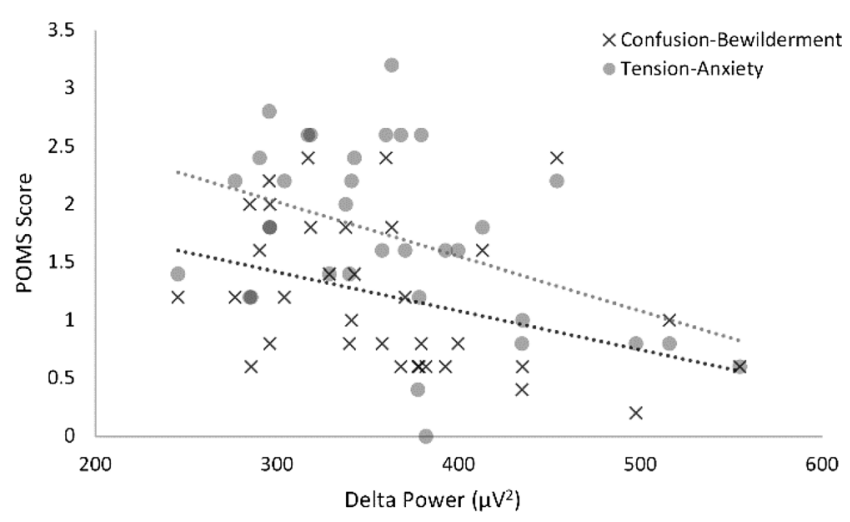

Figure 4. Scatter plot of the relationships between delta power and tension-anxiety, as well as confusion-bewilderment scales in the depression group. POMS $=$ Profile of Mood States.

decreased slow frequency activity. Specifically, positive correlations were detected between restfulness upon awakening with delta $(r=.465, p=.02)$ and theta $(r=.613, p=.001)$ activity across the night. Reported sleep quality in the morning also increased with delta $(r=.408, p=.04)$ and theta $(r=.439, p=.03)$ activity across the night (see Figure 5).

\section{Discussion}

This study aimed to (a) characterize the respective roles of slow and fast frequency activity during sleep in depression, (b) explore the relationship between brain physiology during sleep with

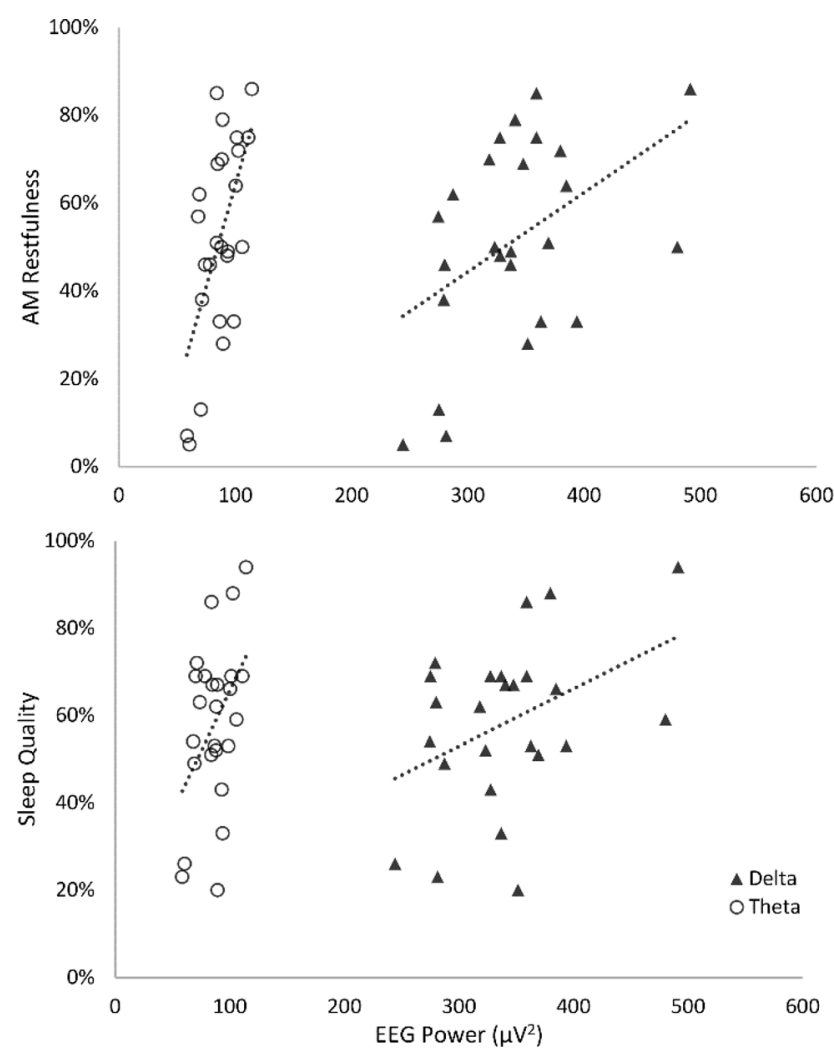

Figure 5. Scatter plots of the relationships between delta and theta power with reported (a) restfulness in the morning, and (b) sleep quality. 
depression symptom and mood profiles, and (c) examine sex as a moderator in the relationship between sleep and depression. It has been posited that sleep difficulties in depression may be related to either abnormalities in sleep homeostasis (i.e., poor sleep due to impaired sleep drive; e.g., Borbély et al., 1984) or cortical hyperarousal as indexed by intrusions of high frequency activity (e.g., Perlis et al., 2001). Furthermore, while sex differences have been robustly documented in sleep and depression (Armitage \& Hoffmann, 2001), very few studies have examined sex as a moderator in the context of both slow and fast frequency activity. To the best of our knowledge, this is the first study to examine both slow and fast frequency in depression with sex as a moderator, particularly in a large sample of clinically validated depressed individuals. Evidence indicate that, while depression is generally characterized by reduced activity in the slow frequency range, females in particular may be prone to intrusion of fast frequency activity that is related to increased symptom severity. Results also suggest that decreased slow frequency activity is related to poorer mood (increased anxiety and confusion).

The reduction of slow frequency activity in depression found in this study, particularly in the earlier portion of the night, is likely associated with abnormalities in sleep homeostasis. Prior research has proposed that depressed individuals may experience a deficit in building homeostatic pressure during wakefulness, thereby resulting in reduced slow-wave activity during sleep. Another recent study by the authors demonstrated that, relative to healthy individuals, depressed participants, particularly if they are male, show a dampened slow-wave activity response to a 3-h delay challenge to sleep homeostasis (Goldschmied et al., 2014). Slow-wave activity is associated with restorative sleep, and occurs more densely toward the beginning of the night. The lack of restorative sleep in depression in turn impacts neuronal functioning in the daytime. This is also consistent with the synaptic homeostasis hypothesis (Tononi \& Cirelli, 2003, 2006), which states that reduced slowwave activity leads to inadequate synaptic depotentiation during sleep, and therefore synaptic overload upon awakening. Consequences of synaptic overload include reductions in neuronal excitability, increased synaptic failure, and reduced plasticity. Not surprisingly, these neuronal consequences also map onto symptoms characteristic of depression, such as fatigue, anhedonia, impaired attention and concentration, and decreased motivation. Results of the present study also indicate that decreased slow frequency activ- ity is associated not only with reduced restfulness upon awakening, but also with increased negative mood and depression severity.

Results from this study also suggest that females in particular may be more likely to experience fast frequency activity related to increased depression severity. Fast frequency activity is likely indicative of cortical arousal, such as worry or rumination (Perlis, Giles, Mendelson, Bootzin, \& Wyatt, 1997). Rumination has been robustly implicated in the etiology of depression for females (for review, see Nolen-Hoeksema, 1990), which may explain why fast frequency activity in this study was associated with depression severity specifically in females. This finding is also consistent with previous research showing increased incidence and amplitude of beta activity in a small sample of depressed females (Armitage et al., 1995). Although only approaching significance, results in this study also showed that decreased beta activity was related to increased anxiety, which may appear inconsistent with the previous result. However, it should be noted that the smaller sample size that included mood data precluded a meaningful sex-specific analysis, and therefore included both males and females, which may explain the seemingly inconsistent finding. Furthermore, it may be possible that the relationship between beta activity and mood may not be linear, such that only excessive beta activity beyond a certain threshold may be related to anxious mood.

Limitations in this study include the use of archival data, which were not acquired for the purpose of the specific analyses conducted in this study. However, standardized methodology was used across all studies from which the samples were derived. Another limitation of using archival data includes the use of data collection methodologies that have since been updated, such as the use of linked-ear reference (Miller, Lutzenberger, \& Elbert, 1991; Nunez, Pilgreen, Westdorp, Law, \& Nelson, 1991).

\section{Conclusions}

Results suggest that sleep in depression may be characterized by an overall decrease in slow-wave activity, which is related to elevated anxious and depressed mood the following morning. The decrease in activity is more prominent in the slower frequencies, indicating that this may be related to a brain deficit in achieving restorative sleep. Furthermore, sex differences were detected, with only females showing a relationship between depression severity and fast frequency activity.

\section{References}

Armitage, R. (2007). Sleep and circadian rhythms in mood disorders. Acta Psychiatrica Scandinavica, 115, 104-115.

Armitage, R., \& Hoffmann, R. (1997). Sleep electrophysiology of major depressive disorders. Current Review of Mood and Anxiety Disorders, 1, 139-151.

Armitage, R., \& Hoffmann, R. (2001). Sleep EEG, depression and gender. Sleep Medicine Reviews, 5, 237-246.

Armitage, R., Hoffmann, R., Fitch, T., Trivedi, M., \& Rush, A. (2000). Temporal characteristics of delta activity during NREM sleep in depressed outpatients and healthy adults: Group and sex effects. Sleep, 23, 607-617.

Armitage, R., Hudson, A., Trivedi, M., \& Rush, A. (1995). Sex differences in the distribution of EEG frequencies during sleep: Unipolar depressed outpatients. Journal of Affective Disorders, 34, 121-129.

Barnett, K. J., \& Cooper, N. J. (2008). The effects of a poor night sleep on mood, cognitive, autonomic and electrophysiological measures. Journal of Integrative Neuroscience, 7, 405-420.

Borbély, Tobler, I., Loepfe, M., Kupfer, D. J., Ulrich, R. F., Grochocinski, V., ... Matthews, G. (1984). All-night spectral analysis of the sleep
EEG in untreated depressives and normal controls. Psychiatry Research, 12, 27-33.

Dolsen, M. R., Asarnow, L. D., \& Harvey, A. G. (2014). Insomnia as a transdiagnostic process in psychiatric disorders. Current Psychiatry Reports, 16, 471. Retrieved from http://doi.org/10.1007/s11920-014-0471-y

Faul, F., Erdfelder, E., Lang, A., \& Buchner, A. (2007). G* Power 3: A flexible statistical power analysis program for the social, behavioral, and biomedical sciences. Behavior Research Methods, 39, 175-191.

First, M., Spitzer, R., Gibbon, M., \& Willams, J. (2002). Structured clinical interview for DSM-IV-TR axis I disorders, research version, patient edition. New York, NY: Biometrics Research, New York State Psychiatric Institute.

Frey, S., Birchler-Pedross, A., Hofstetter, M., Brunner, P., Götz, T., Münch, M., ... Cajochen, C. (2012). Young women with major depression live on higher homeostatic sleep pressure than healthy controls. Chronobiology International, 29, 278-294.

Goldschmied, J. R., Cheng, P., Armitage, R., \& Deldin, P. J. (2014). Examining the effects of sleep delay on depressed males and females and healthy controls. Journal of Sleep Research, 23, 664-672. 
Hall, M., Buysse, D. J., Nowell, P. D., Nofzinger, E. A., Houck, P., Reynolds, C. F., \& Kupfer, D. J. (2000). Symptoms of stress and depression as correlates of sleep in primary insomnia. Psychosomatic Medicine, 62, 227-230.

Hamilton, M. (1967). Development of a rating scale for primary depressive illness. British Journal of Social and Clinical Psychology, 6, 278-296.

Harvey, A. G. (2008). Insomnia, psychiatric disorders, and the transdiagnostic perspective. Current Directions in Psychological Science, 17, 299-303.

Manber, R., Edinger, J. D., Gress, J. L., San Pedro-Salcedo, M. G., Kuo, T. F., \& Kalista, T. (2008). Cognitive behavioral therapy for insomnia enhances depression outcome in patients with comorbid major depressive disorder and insomnia. Sleep, 31, 489-495.

McNair, D. M., Lorr, M., \& Droppleman, L. F. (1971). POMS: Profile of mood states. San Diego, CA: Educational and Industrial Testing Service.

Mendelson, W. B., James, S. P., Garnett, D., Sack, D. A., \& Rosenthal, N. E. (1986). A psychophysiological study of insomnia. Psychiatry Research, 19, 267-284.

Mendelson, W. B., Sack, D. A., James, S. P., Martin, J. V., Wagner, R., Garnett, D., ... Wehr, T. A. (1987). Frequency analysis of the sleep EEG in depression. Psychiatry Research, 21, 89-94.

Miller, G. A., Lutzenberger, W., \& Elbert, T. (1991). The linked-reference issue in EEG and ERP recording. Journal of Psychophysiology, 5, 273-276.

Nofzinger, E. A., Nowell, P. D., Buysse, D. J., Vasco, R. C., Thase, M. E., Frank, E., ... Reynolds, C. F. (1999). Towards a neurobiology of sleep disturbance in primary insomnia and depression: A comparison of subjective, visually scored, period amplitude, and power spectral density sleep measures [Supplement 1]. Sleep, 22, S99-S99.

Nolen-Hoeksema, S. (1990). Sex differences in depression. Stanford, CA: Stanford University Press.

Nunez, P. L., Pilgreen, K. L., Westdorp, A. F., Law, S. K., \& Nelson, A. V. (1991). A visual study of surface potentials and Laplacians due to distributed neocortical sources: Computer simulations and evoked potentials. Brain Topography, 4, 151-168.

Perlis, M. L., Giles, D. E., Mendelson, W. B., Bootzin, R. R., \& Wyatt, J. K. (1997). Psychophysiological insomnia: The behavioural model and a neurocognitive perspective. Journal of Sleep Research, 6, 179188.

Perlis, M. L., Giles, D. E., Buysse, D. J., Thase, M. E., Tu, X., \& Kupfer, D. J. (1997). Which depressive symptoms are related to which sleep electroencephalographic variables? Biological Psychiatry, 42, 904-913.
Perlis, M. L., Merica, H., Smith, M. T., \& Giles, D. E. (2001). Beta EEG activity and insomnia. Sleep Medicine Reviews, 5, 365-376.

Plante, D. T., Landsness, E. C., Peterson, M. J., Goldstein, M. R., Riedner, B. A., Wanger, T., ... Benca, R. M. (2012). Sex-related differences in sleep slow wave activity in major depressive disorder: A high-density EEG investigation. BMC Psychiatry, 12, 146.

Rechtschaffen, A., \& Kales, A. (1968). A manual of standardized terminology, techniques, and scoring systems for sleep stages of human subjects. Washington, DC: Superintendent of Documents, U.S. Government Printing Office.

Reynolds, C., Frank, E., Houck, P. R., Mazumdar, S., Dew, M. A., Cornes, C., ... Kupfer, D. J. (1997). Which elderly patients with remitted depression remain well with continued interpersonal psychotherapy after discontinuation of antidepressant medication? American Journal of Psychiatry, 154, 958-962.

Reynolds, C., \& Kupfer, D. J. (1987). Sleep research in affective illness: State of the art circa 1987. Sleep: Journal of Sleep Research \& Sleep Medicine, 10, 199-215.

Reynolds, C., Kupfer, D. J., Thase, M. E., Frank, E., Jarrett, D. B., Coble, P. A., ... Houck, P. R. (1990). Sleep, gender, and depression: An analysis of gender effects on the electroencephalographic sleep of 302 depressed outpatients. Biological Psychiatry, 28, 673-684.

Riemann, D., Spiegelhalder, K., Feige, B., Voderholzer, U., Berger, M., Perlis, M., \& Nissen, C. (2010). The hyperarousal model of insomnia: A review of the concept and its evidence. Sleep Medicine Reviews, 14, 19-31.

Stepanski, E., Zorick, F., Roehrs, T., \& Young, D. (1988). Daytime alertness in patients with chronic insomnia compared with asymptomatic control subjects. Sleep: Journal of Sleep Research \& Sleep Medicine, $11,54-60$.

Tononi, G., \& Cirelli, C. (2003). Sleep and synaptic homeostasis: A hypothesis. Brain Research Bulletin, 62, 143-150.

Tononi, G., \& Cirelli, C. (2006). Sleep function and synaptic homeostasis. Sleep Medicine Reviews, 10, 49-62.

Vanderlind, W. M., Beevers, C. G., Sherman, S. M., Trujillo, L. T., McGeary, J. E., Matthews, M. D., ... Schnyer, D. M. (2014). Sleep and sadness: Exploring the relation among sleep, cognitive control, and depressive symptoms in young adults. Sleep Medicine, 15, 144-149.

(Received October 23, 2014; Accepted May 29, 2015) 